\title{
Gaugino-Assisted Anomaly Mediation
}

\author{
David Elazzar Kaplan \\ High Energy Physics Division, Argonne National Laboratory, Argonne, IL 60439 \\ Enrico Fermi Institute, Univ. of Chicago, 5640 Ellis Avenue, Chicago, IL 60637 \\ dkaplan@theory.uchicago.edu

\section{Graham D. Kribs} \\ Department of Physics, Carnegie Mellon University, Pittsburgh, PA 15213-3890 \\ Department of Physics, University of Wisconsin, 1150 University Avenue, \\ Madison, WI 53706.* \\ kribs@pheno.physics.wisc.edu
}

ABSTRACT: We present a model of supersymmetry breaking mediated through a small extra dimension. Standard model matter multiplets and a supersymmetry-breaking (or "hidden") sector are confined to opposite four-dimensional boundaries while gauge multiplets live in the bulk. The hidden sector does not contain a singlet and the dominant contribution to gaugino masses is via anomaly-mediated supersymmetry breaking. Scalar masses get contributions from both anomaly mediation and a tiny hard breaking of supersymmetry by operators on the hidden-sector boundary. These operators contribute to scalar masses at one loop and in most of parameter space, their contribution dominates. Thus it is easy to make all squared scalar masses positive. As no additional fields or symmetries are required below the Planck scale, we consider this the simplest working model of anomaly mediation. The gaugino spectrum is left untouched and the phenomenology of the model is roughly similar to anomaly mediated supersymmetry breaking with a universal scalar mass added. We identify the main differences in the spectrum between this model and other approaches. We also discuss mechanisms for generating the $\mu$ term and constraints on additional bulk fields.

KEYworDs: Supersymmetry Breaking, Extra Dimensions, Gaugino Mediation.

\footnotetext{
${ }^{*}$ Present address.
} 


\section{Introduction}

If supersymmetric partners of standard model particles are discovered by the collider experiments of the coming decade, one of the first theoretical questions that must be answered is "how did supersymmetry break?". Softly broken supersymmetry as an effective low energy theory contains over one hundred parameters, but we expect there is an organizing principle that determines most of these parameters. This involves a mechanism to break supersymmetry and a means to communicate, or mediate, supersymmetry breaking to the superpartners of the Standard Model (SM). It behooves us to search for simple and compelling models that generate soft masses of order the weak scale in a predictive and experimentally allowed fashion.

Viable models are those which do not have contributions to processes beyond their experimental bounds. Processes which include, for example, flavor changing neutral currents (FCNC), CP violation or lepton flavor violation, are suppressed in the standard model, and thus whatever generates the soft parameters must also sufficiently suppress flavor and CP violation[1, 2]. The former is naturally suppressed in models of gauge-mediated supersymmetry breaking (GMSB), where soft terms come from loop contributions involving standard model gauge interactions [3]. Since the gauge interactions are flavor blind, the soft masses are as well.

Another candidate mechanism for mediating supersymmetry breaking is via the superconformal anomaly [4, 5, 6]. In the absence of pure singlets in the supersymmetry breaking sector, gaugino masses are generated at the one-loop level [5]. If in addition the standard model fields are confined to a four-dimensional boundary in a higherdimensional space and supersymmetry breaking occurs on a different boundary (the hidden sector) spatially separated from the standard model, then the dominant contribution to scalar masses come from anomaly mediation and are of the same order as the gaugino masses [4]. Large contributions to FCNC processes are avoided because the scalar masses, in particular the first and second generation, are dominated by contributions proportional to the beta functions of gauge couplings. Similarly, CP violation can also be suppressed if all weak scale masses are generated by a single hidden sector parameter.

However, pure anomaly mediation predicts tachyonic sleptons. The squared scalar mass contributions are proportional to beta functions of gauge couplings, with nonasymptotically free gauge groups giving them negative contributions. In the minimal supersymmetric standard model (MSSM) the $\mathrm{SU}(2) \times \mathrm{U}(1)$ gauge groups are nonasymptotically free, and thus the squared masses of sleptons (in particular, those of the first two generations) have overwhelmingly dominant contributions which are negative. 
Clearly, additional model building is required. The task is nontrivial since the form of the soft parameters are renormalization group invariant, depending only on the infrared values of the beta functions 4 , 5].

Nevertheless, interesting solutions which avoid these difficulties have been suggested. Pomarol and Rattazzi proposed adding a light singlet to the visible sector which obtains a large vacuum expectation value (vev) for its scalar component, and then used this scale to generate a threshold that does not decouple [7]. Thus a spectrum very different from that of the original anomaly-mediated models is produced \&8 and the slepton mass problem can be solved. This idea has also been applied to a GUT threshold in Ref. [9]. Katz, Shadmi, and Shirman showed that threshold effects do not decouple at higher orders in the supersymmetry breaking order parameter, but this effect is only significant for thresholds that are not too far above the weak scale [10]. Another possibility is to alter the infrared structure of the MSSM directly, by adding new matter charged under the standard model and new Yukawa couplings [11] or an additional U(1) gauge group [7, 10, 12, 13]. Finally, one can add additional non-MSSM fields to the bulk to generate new contributions to soft masses [14, 15, 16], though in most cases, these contributions dominate those of anomaly mediation. In any case, the additional structure needed to solve the slepton mass problem invariably affects much more of the sparticle spectrum than just the slepton masses, and so the phenomenology need not have any resemblance to "pure" anomaly mediation.

In this article, we present a simple model of supersymmetry breaking mediated by both supergravity and MSSM gauge multiplets that live in the higher dimensional "bulk". The hidden sector is spatially separated from the matter sector, and consequently contact interactions are forbidden by locality. In this way, the model is similar to gaugino-mediated supersymmetry breaking ( $\tilde{\mathrm{g} M S B}$ ] 15, 16] as well as anomaly mediation [4, 5]. However, unlike the original gaugino mediation proposals [15, 16], we assume there are no fundamental singlets in the hidden sector. This is well motivated as most known models of dynamical supersymmetry breaking lack singlets [17, 18], and was a major motivation that led to uncovering anomaly-induced gaugino masses [5]. We will show that this implies the dominant contribution to gaugino masses is from anomaly mediation. Scalar masses also receive their usual contribution from anomaly mediation, but in this model the gauge and gaugino fields can also communicate supersymmetry breaking to the matter sector. We will show that the leading gaugino-mediated contribution comes from a set of higher dimensional operators which generically appear on the hidden sector boundary. These operators result in tiny corrections to the wave function renormalization of gauginos different from gauge fields and thus are a hard breaking of supersymmetry. These operators contribute to squared 
scalar masses at one loop. In doing a full five-dimensional calculation, we show that their contributions are of the right size to solve the slepton mass problem of anomaly mediation. Below the compactification scale, the model therefore has all the appearances of anomaly mediation with an additional contribution to scalar masses. This is perhaps the most minimal solution to the slepton mass problem in anomaly mediation. Notice that because the interactions that give rise to the additional scalar mass contributions involve gauge and gaugino fields, they are automatically flavor-blind. We need only assume that the extra dimension is large enough such that the exponentially suppressed wave-function overlap estimates of contact FCNC interactions are indeed suppressed without fine tuning (a compactification length one order of magnitude larger than the five-dimensional Planck length is sufficient).

The rest of this paper is organized as follows. In Section 2 we review the salient features of anomaly mediation including the predicted spectrum and its decoupling features. In Section 3 we present our model. We show that the dominant contribution to gaugino masses is from anomaly mediation, while the bulk gauge and gaugino fields give rise to additional contributions to scalar masses. In Section 1 we discuss phenomenology, including the list of input parameters and the generic spectrum. In Section 5 we briefly discuss solving the $\mu$ problem and the effects of adding bulk fields and in Section 6 we conclude. An appendix is included in which we demonstrate how we take into account the effects of GUT fields at the threshold.

\section{Anomaly mediated supersymmetry breaking}

In this section we review the contributions to soft terms from the superconformal anomaly [4, 5] as well as the construction in extra dimensions which makes these contributions the dominant ones [4]. There are actually several distinct contributions to, for instance, gaugino masses from the anomalous symmetries in the supergravity Lagrangian [6] (see also [19]). These contributions reduce to that of just the superconformal anomaly 4 , 5] when it is assumed that all vacuum expectation values in or coupled to the hidden sector are much smaller than the Planck mass, $M_{\mathrm{Pl}}$. For the remainder of this paper, we shall make this assumption.

If singlets are absent from the hidden sector, the dominant contribution to gaugino masses comes from anomaly mediation and is loop suppressed with respect to the gravitino mass, $m_{3 / 2}$. Scalar masses would in general be of order $m_{3 / 2}$ as usual in "SUGRA" scenarios, meaning that not only does one still have the usual FCNC problem, but one must also fine-tune the coefficients of at least some of the operators to be of order a loop factor [5]. However, scalar masses can be suppressed by spatially separating the 
hidden sector from the MSSM in extra dimensions. For our purposes, we imagine one extra flat spatial dimension compactified on $S^{1} / Z_{2}$. The fifth coordinate $y$ runs from $-L$ to $L$ with $y \rightarrow-y$ identified. The four-dimensional hypersurfaces at $y=0$ and $L$ are where the MSSM and hidden sectors live respectively, while supergravity lives in the bulk [4]. The compact dimension could be stabilized by the mechanisms in Ref. [20] or 21] without greatly changing the dynamics of supersymmetry breaking.

With an extra dimension of size $L$, the five-dimensional Planck scale $M_{*}$ is related to the four-dimensional (reduced) Planck scale $M_{\mathrm{Pl}}$ by [22]

$$
M_{*}^{3} L=M_{\mathrm{Pl}}^{2}
$$

To solve the flavor problem, we must suppress flavor violating operators such that the off-diagonal squared squark masses are at most of order $10^{-3}$ times the diagonal squared squark masses [2]. Thus, operators like

$$
\frac{1}{M_{*}^{2}} \int d^{4} \theta \Sigma^{\dagger} \Sigma Q_{i}^{\dagger} Q_{j} \quad(i \neq j)
$$

should conservatively have a coefficient less than of order $10^{-3} \times\left(m_{\text {weak }}^{2} / m_{3 / 2}^{2}\right) \sim 10^{-7}$. These operators could be generated by the exchange of particles of mass $M_{*}$ and thus are suppressed by the exponential suppression of the Yukawa potential $e^{-M_{*} L}$, requiring $M_{*} L \gtrsim 16$.

To calculate the soft masses, it is simplest to use the compensator formalism of supergravity [23], which has been discussed in the original anomaly mediation papers 44, 5. In this approach, superconformal symmetry is made manifest by using the conformal compensator $\Phi$ as a spurion for symmetry breaking, where

$$
\Phi=1+\theta^{2} m_{3 / 2}
$$

In this formalism, $\Phi$ is the only source of supersymmetry breaking in the visible sector. The coupling of the MSSM to supergravity can be written

$$
\mathcal{L}=\int d^{4} \theta \Phi^{\dagger} \Phi f\left(Q^{\dagger}, e^{-V} Q\right)+\int d^{2} \theta \Phi^{3}\left(W(Q)+\frac{1}{g^{2}} \mathcal{W}_{\alpha} \mathcal{W}^{\alpha}\right)+\text { h.c. }+\ldots
$$

where we have written the Kähler potential, superpotential, and gauge superfield terms in flat space. Each superfield can be rescaled by $\Phi^{-n}$ where $n$ is the canonical dimension of the field. Thus, in the MSSM without a $\mu$ term, supersymmetry breaking does not appear at tree level. However, all dimensionful parameters will come with the appropriate power of $\Phi$. The key observation is that conformal symmetry is broken at 
the loop level by any regulator of the theory, and that the cutoff scale must also appear with the compensator.

Thus, we expect soft masses to appear at loop level. These masses can be obtained via explicit calculation with a regulator [4, 5] or using the method of 'analytical continuation into superspace' [24, 5, 7]. In any case, one finds 4, 5]

$$
\begin{aligned}
M_{a} & =\frac{\beta_{g_{a}}}{g_{a}} m_{3 / 2} \\
\tilde{m}_{i}^{2} & =-\frac{1}{4}\left(\frac{\partial \gamma_{i}}{\partial g} \beta_{g}+\frac{\partial \gamma_{i}}{\partial Y} \beta_{Y}\right) m_{3 / 2}^{2} \\
A_{Y} & =-\frac{\beta_{Y}}{Y} m_{3 / 2}
\end{aligned}
$$

There are three important aspects of this spectrum to notice. First, flavor violation is proportional to the Yukawa couplings. Second, because the beta functions for SU(2) and $\mathrm{U}(1)$ are both positive, the squared masses of at least the first two generations of sleptons are negative. Third, the forms of these soft masses are renormalization group invariant to all orders [5, 25]. In fact, supersymmetric mass thresholds that are significantly above the weak scale completely decouple [5, 7, 10]. This can be seen as arising from a cancellation between the anomaly-induced supersymmetry breaking contributions with threshold contributions. Hence, the results in Eqs. (2.5) are valid at the weak scale. This presents a significant obstacle in solving the slepton mass problem.

The simplest approach to dealing with the slepton mass problem is to simply add an additional universal scalar mass squared $m_{0}^{2}$ to the spectrum [26, 27]. This is really just a phenomenological fix, however, and levies the explanation of why FCNC are suppressed into the postulate that $m_{0}^{2}$ is flavor-diagonal. Nevertheless, this does allow an analysis of the phenomenology of gaugino masses proportional to the gauge beta functions.

As discussed in the introduction, there have been several groups that have specific proposals for solving the slepton mass problem. In all cases, the goal is to circumvent the decoupling phenomenon. All of these proposals require the addition of additional matter and/or gauge groups to the MSSM and all but those in [12, 13] significantly change the gaugino spectrum ${ }^{\dagger}$.

Thus, anomaly mediation by itself is a fascinating but phenomenologically unacceptable means to communicate supersymmetry breaking. Any model in which the

\footnotetext{
${ }^{\dagger}$ Adding weak-scale Fayet-Illiopoulos terms for hypercharge and an additional U(1) in the MSSM does not affect the gaugino spectrum [12, 13] and thus a model in which these terms are generated dynamically at the correct size would be quite interesting.
} 
AMSB contributions are important (or dominant) must be supplemented by a true solution to the slepton mass problem, and this generally requires rather complicated additional structure that need not leave the spectrum looking anything like simply adding a universal scalar mass.

\section{Gaugino assisted anomaly mediation}

Consider taking the model of anomaly mediation in its original form [4], but place the MSSM gauge and gaugino fields in the bulk. If singlets in the hidden sector exist, the dominant contribution to soft masses comes from gaugino mediation [15, 16], since the anomaly contributions are loop suppressed. Now, consider the scenario in which no singlets exist on the hidden sector boundary. Singlets are absent in many models of dynamical supersymmetry breaking, and so this can be thought of as a well-motivated "special case" of gaugino mediation. We shall show below that, in this case, anomaly mediation plays a much more important role, but that the presence of gauge and gaugino fields in the bulk results in additional contributions to scalar masses that are naturally of the same size as their anomaly-mediated counterparts. The result is an explicit model in which anomaly-mediated contributions are large (and dominant for the gaugino masses), while the slepton masses can easily be positive.

There are a few subtleties of placing gauge and gaugino fields in $4+1$ dimensions. The vector multiplet has twice as many physical degrees of freedom. The gauge field $A^{\mu}$ has a fifth component $A^{5}$, there are two 2-component gaugino spinors, $\lambda^{1}$ and $\lambda^{2}$, and there is a real scalar field $\Phi$, all in the adjoint representation. We can selectively decouple the effects of some of these fields by making them odd under the $Z_{2}$ part of the compactification, explicitly breaking half of the $(N=2$ in four dimensions) supersymmetries at the boundary. Then, the contributions of these fields to what follows are negligible. For a detailed prescription and analysis, see Ref. 28].

Putting gauge fields in the bulk allows for local operators combining the gauge multiplets with fields of the hidden sector. For instance, if the hidden sector contains a singlet $S$, gaugino masses are generated by the operator

$$
\int d^{2} \theta \frac{S}{M_{*}^{2}} W^{\alpha} W_{\alpha} \delta(y-L)
$$

which gives $M_{1 / 2}=F_{S} /\left(M_{*}^{2} L\right)=\left(F_{S} / M_{\mathrm{Pl}}\right) \times\left(M_{*} L\right)^{-1 / 2}$. This can be compared with the anomaly-mediated contribution for a gravitino mass $m_{3 / 2} \equiv F / M_{\mathrm{Pl}}$ where we associate the fundamental $F$ term with $F_{S}$, resulting in $M_{1 / 2}^{\mathrm{AM}}=\left(16 \pi^{2}\right)^{-1} F_{S} / M_{\mathrm{Pl}}$. For 
$M_{*} L \ll 10^{4}$ (which is always satisfied if $M_{*}$ is the scale where either the gauge or gravitational coupling gets strong), the gaugino-mediated contribution dominates, and the result is gaugino mediated supersymmetry breaking [15, 16]. Scalar masses are then generated at the weak scale via the renormalization group and the spectrum looks similar to that of no-scale models [29], with differences depending on the compactification scale and how the $\mu$ term is generated [15, 16, 30, 31, 32].

If we now suppose that there are no singlets in the hidden sector, the operator (3.1) is absent. In the Kähler potential, gaugino masses can be generated but they are highly suppressed. The leading higher-dimension operator with the least powers of $M_{*}$ is

$$
\int d^{4} \theta \frac{\Sigma^{\dagger} \Sigma}{M_{*}^{4}} W^{\alpha} W_{\alpha} \delta(y-L) .
$$

where $\Sigma$ is a hidden sector field charged under some hidden sector group(s) with the largest auxiliary component vev $F_{\Sigma}$. However, it is obvious that this just gives

$$
\frac{F_{\Sigma}^{\dagger}}{M_{*}^{2}} \int d^{2} \theta \frac{\Sigma}{M_{*}^{2}} W^{\alpha} W_{\alpha} \delta(y-L)
$$

and so takes the same form as (3.1) except that there is the additional suppression of $F / M_{*}^{2}$. Hence, the contribution arising from this operator is of order $F^{2} /\left(M_{*}^{4} L\right)$, which is negligible compared with the anomaly-mediated contribution.

However, even though (3.1) is not allowed, there are a number of operators expected to appear on the hidden sector boundary which can contribute to the spectrum. Some allowed operators are

$$
\begin{array}{ll}
\int d^{4} \theta & \frac{\Sigma^{\dagger} D^{2} \Sigma}{M_{*}^{5}} W^{\alpha} W_{\alpha} \delta(y-L), \\
\int d^{4} \theta & \frac{\Sigma^{\dagger} \Sigma}{M_{*}^{5}} W^{\alpha} D^{2} W_{\alpha} \delta(y-L), \\
\int d^{4} \theta & \frac{\Sigma^{\dagger} \Sigma}{M_{*}^{5}} D_{\beta} W^{\alpha} D^{\beta} W_{\alpha} \delta(y-L), \\
\int d^{4} \theta & \frac{\Sigma^{\dagger} D_{\beta} \Sigma}{M_{*}^{5}} W^{\alpha} D^{\beta} W_{\alpha} \delta(y-L), \\
\int d^{4} \theta & \frac{\Sigma^{\dagger} \Sigma}{M_{*}^{5}} D^{\beta} W^{\alpha} D^{\alpha} W_{\beta} \delta(y-L), \\
\text { etc... }
\end{array}
$$


where $D_{\alpha}$ is the superspace derivative and the ellipsis denotes additional terms similar to those above. When $\Sigma$ is replaced with its $F$ component, these operators become tiny corrections to the kinetic terms of the gauge multiplet. For instance, Eq. (3.4) becomes

$$
\begin{aligned}
\int d^{4} \theta & \frac{\Sigma^{\dagger} D^{2} \Sigma}{M_{*}^{5}} W^{\alpha} W_{\alpha} \delta(y-L) \\
& \rightarrow \int d^{2} \theta \frac{F_{\Sigma}^{\dagger} F_{\Sigma}}{M_{*}^{4}\left(M_{*} L\right)} W^{0 \alpha} W_{\alpha}^{0} \\
& \rightarrow \frac{F_{\Sigma}^{\dagger} F_{\Sigma}}{M_{*}^{4}\left(M_{*} L\right)}\left[-2 i \lambda \sigma^{\mu} D_{\mu} \bar{\lambda}-\frac{1}{2} F^{\mu \nu} F_{\mu \nu}+D^{2}+\frac{i}{4} F^{\mu \nu} \widetilde{F}_{\mu \nu}\right]
\end{aligned}
$$

where the five-dimensional supersymmetric field strength $W^{\alpha}$ has been replaced by its (rescaled) zero mode $W^{0 \alpha}$, and $F^{\mu \nu}, \lambda$ and $D$ are the four-dimensional gauge field strength, gaugino and auxiliary components respectively. Thus the effect of this operator is to simply rescale the gauge coupling by a tiny amount.

The other operators in Eqs. (3.5)-(3.9), however, have non-negligible effects. For example, the operator Eq. (3.5) becomes [15]

$$
\int d^{4} \theta \frac{\Sigma^{\dagger} \Sigma}{M_{*}^{5}} W^{\alpha} D^{2} W_{\alpha} \delta(y-L) \rightarrow \frac{\left|F_{\Sigma}\right|^{2}}{M_{*}^{4}} \frac{1}{\left(M_{*} L\right)} \lambda \sigma^{\mu} D_{\mu} \bar{\lambda},
$$

where $\lambda$ is the four-dimensional zero-mode of the gaugino. Note that this operator contributes only to the gaugino kinetic term. This corresponds to a wave-function renormalization of the gaugino slightly different from that of the gauge field and auxiliary components and thus leads to a (albeit tiny) hard breaking of supersymmetry.

First, let us make a rough estimate of the effects of this hard breaking on the spectrum. To do this, we rescale the gaugino wave function such that its kinetic term is canonical at the compactification scale $L^{-1}$. Such a rescaling changes (for example) the gaugino-quark-squark coupling $g_{\tilde{g} q \tilde{q}}$ relative to the gauge coupling $g$ as $^{\ddagger}$

$$
g_{\tilde{g} q \tilde{q}} \rightarrow g_{\tilde{g} q \tilde{q}}^{\prime} \simeq\left(1+\frac{F_{\Sigma}^{2}}{M_{*}^{5} L}\right) g .
$$

Now the one-loop calculation of the contribution to squared scalar masses appears quadratically divergent. Taking $\Lambda=L^{-1}$ to be the cutoff, we can approximate this contribution as

$$
\delta m_{\tilde{f}}^{2} \sim \frac{g^{2}}{16 \pi^{2}} \frac{\left|F_{\Sigma}\right|^{2}}{M_{*}^{5} L} \Lambda^{2}
$$

\footnotetext{
${ }^{\ddagger}$ A shift of the gaugino coupling relative to the gauge coupling also arises from superoblique corrections [33], but this is completely distinct from the operators we are adding.
} 


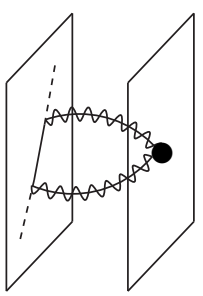

(a)

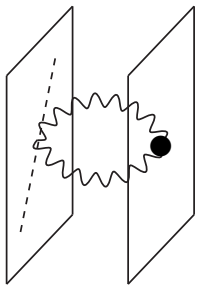

(b)

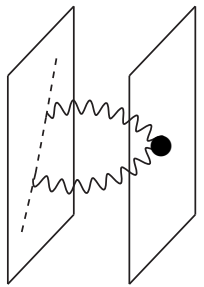

(c)

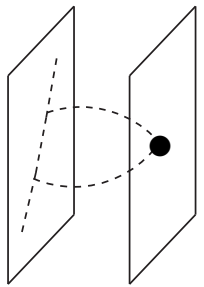

(d)

Figure 1: One loop contributions to scalar masses. The dot represents the operator insertion in the five-dimensional propagators. The five-dimensional propagators in the loops are (a) gauginos, (b) \& (c) gauge bosons, and (d) the real scalar adjoint $\Phi$.

$$
\sim \frac{g^{2}}{16 \pi^{2}} \frac{\left|F_{\Sigma}\right|^{2}}{M_{\mathrm{Pl}}^{2}} \frac{1}{\left(M_{*} L\right)^{2}}
$$

From this estimate it is clear that there is some range of compactification scales $L^{-1}$ for which this contribution is as or more important as the one from anomaly mediation. Notice that these contributions ought to be present in any effective theory. In particular, in ordinary supergravity models we can write the operator

$$
\int d^{4} \theta \frac{\Sigma^{\dagger} \Sigma}{M_{\mathrm{Pl}}^{4}} W^{\alpha} D^{2} W_{\alpha}
$$

(where now the field strength superfield $W^{\alpha}$ is purely four-dimensional) which also leads to contributions to scalar masses of order $F^{2} / M_{*}^{2}$ times a loop factor.

Now we examine this new scalar mass contribution more carefully. The full oneloop calculation is extremely well approximated by a five-dimensional loop diagram with a single insertion of the operator (3.11) on the gaugino propagator (see Fig. 17a). Using the mixed momentum/position space propagators $\mathcal{P}$ as defined in the Appendix (see also Ref. 28, 34, 15]), we have

$$
\tilde{m}_{i}^{2}=\frac{2 g_{(5)}^{2} C(i)}{16 \pi^{2}} \int d^{4} q \operatorname{tr}\left[\frac{-1}{\not q} \mathcal{P}(q ; 0, L) \frac{\not F_{\Sigma}^{2}}{M_{*}^{5}} \mathcal{P}(q ; L, 0)\right]
$$

where $g_{(5)}$ is the five-dimensional gauge coupling, $C(i)$ is the quadratic Casimir for the $i$ matter scalar representation, and the integral is over all (Euclidean) four-momenta. The integrand is highly peaked around the compactification scale so when computing the spectrum, it is reasonable to begin renormalization group evolution at $L^{-1}$. The integral over momenta is finite, due to physical point-splitting, and can be done analytically. 
We obtain

$$
\tilde{m}^{2}=2 \zeta(3) \Gamma(4) C(i) \frac{g_{(5)}^{2}}{16 \pi^{2}} \frac{F_{\Sigma}^{2}}{M_{*}^{5} L^{4}}
$$

There are two things to notice about this result. First, there is a numerical enhancement of the coefficient partially due to the sum of Kaluza-Klein (KK) modes. Second, the form of the contribution Eq. (3.16) looks similar to that of gauge mediated supersymmetry breaking for scalars [3]. The main difference is that the above contribution comes at one-loop with $g^{2} / 16 \pi^{2}$ and not two loops with $\left(g^{2} / 16 \pi^{2}\right)^{2}$. The gauge couplings, however, are unified at this scale and thus the ratio of squared scalar masses $\tilde{m}_{i}^{2}$ and $\tilde{m}_{j}^{2}$ is simply $\sum_{a} C_{a}(i) / \sum_{b} C_{b}(j)$ as it would be for gauge mediation at the GUT scale.

Using $m_{3 / 2}=F_{\Sigma} / M_{\mathrm{Pl}}$, we can rewrite the expression for the scalar masses in Eq. (3.16) in terms of the gravitino mass

$$
\tilde{m}^{2}=2 \zeta(3) \Gamma(4) C(i) \frac{g^{2}}{16 \pi^{2}} \frac{1}{\left(M_{*} L\right)^{2}} m_{3 / 2}^{2}
$$

As predicted in our earlier estimate, this "gaugino-assisted" contribution to the scalar masses is suppressed by one loop factor and two volume factors $M_{*} L$ with respect to $\left(m_{3 / 2}\right)^{2}$. In contrast, the anomaly-mediated contribution is simply two-loop suppressed. If the operator in Eq. (3.11) has a coefficient of order one, the two contributions are of the same order if $\left(M_{*} L\right)^{-2} \sim g^{2} /\left(16 \pi^{2}\right)$. In this case, the slepton mass problem of AMSB is solved. We need only require that the sign of the operator in Eq. (3.11) is such that the contribution to scalar (mass) ${ }^{2}$ is positive. Since we are simply writing the effective operators, the coefficient, including the sign, is undetermined.

All of the operators in Eqs. (3.5)-(3.9) give contributions to scalar masses of the same form as in Eq. (3.17). The operator Eq. (3.6) contributes to both $\left(F_{\mu \nu}\right)^{2}$ and $D^{2}$ terms $^{\S}$. The $\left(F_{\mu \nu}\right)^{2}$ term contributes through the diagrams in Fig. 国(b) and 1(c). In the five-dimensional theory, the $D^{2}$ term is converted to $\left(X^{3}-\partial_{y} \Phi\right)^{2}$, where $X^{3}$ and $\Phi$ are real auxiliary and scalar components of the five-dimensional vector multiplet, respectively [28]. This replacement reveals the fourth diagram, Fig. 1(d). Using the scalar propagator in Ref. [28], we get

$$
\delta m_{\tilde{f}}^{2}=2 \frac{g_{(5)}^{2} F_{\Sigma}^{2}}{M_{*}^{5}} C(i) \int \frac{d^{4} q}{\left(2 \pi^{4}\right)}\left(\frac{1}{L} \sum_{n=-\infty}^{\infty} \frac{q_{5}^{2}}{q^{2}+q_{5}^{2}} \cos q_{5} L\right)^{2} \frac{1}{q^{2}},
$$

\footnotetext{
${ }^{\S}$ We ignore the $F \widetilde{F}$ term as we assume CP is conserved on the hidden sector boundary. This allows us to solve the supersymmetric CP problem [2, 16], but not the strong CP problem.
} 
where $q_{5} \equiv n \pi / L$ and all diagrams are calculated in Euclidean space. The sums in this integral are asymptotic series. They become convergent if we make the assertion

$$
0=\sum_{n}(-1)^{n}=\sum_{n}(-1)^{n} \frac{\left(q^{5}\right)^{2}+q^{2}}{\left(q^{5}\right)^{2}+q^{2}},
$$

which allows us to replace the $\left(k^{5}\right)^{2}$ terms in the numerator with $k^{2}$. This was done in 28] and shown to be a necessary ingredient to preserve supersymmetry. Making this replacement, we get a result of identical form as Eq. (3.16).

The remaining soft parameters to compute are scalar trilinear, or 'A' terms. Scalar trilinear couplings do not get contributions from the operators (3.5)-(3.9) and are nonzero at the compactification scale solely due to anomaly mediation [4, 5]. This is clear since these operators preserve an $R$ symmetry which A terms break. Other operators produce at most negligible contributions.

A few comments about the spectrum are in order. In the four-dimensional description, there is a tower of KK mode copies of the gauge multiplets which all feel supersymmetry breaking from the conformal compensator. Normally we should expect these states to completely decouple as is typical in AMSB. However, it is non-trivial to show that this generically happens as KK masses come directly from dynamics of fields which stabilize the extra dimension. We shall assume that these effects can be made negligible in some cases and leave explicit calculations for future work.

The other threshold one might worry about is that of the GUT scale. If there is a unified theory, there are additional matter fields with GUT scale masses such as Higgs triplets (or the remainder of the Higgs multiplet) or the fields that break the GUT group. These are chiral superfields with a supersymmetric mass of $\sim M_{G U T}$. From the superconformal anomaly, they will get an order weak-scale holomorphic soft mass squared of order $m_{3 / 2} M_{G U T}$. They will also get a non-holomorphic soft mass squared from the one-loop contributions described above. When these fields are integrated out, their holomorphic supersymmetry breaking effects on matter scalar soft masses decouple [由, 5, 7, 11, 10]. The non-holomorphic piece does not contribute to gaugino masses at leading order [35, 36] and as it is of order the weak scale, its contribution to scalars is at least one-loop suppressed compared to the leading contributions .

One impact the GUT fields could have is on the boundary conditions of the MSSM scalars if $L^{-1} \ll M_{\mathrm{GUT}}$. We discuss this possibility in the next section and in the Appendix.

\footnotetext{
"Note, however, that if the GUT scale is generated dynamically through a mechanism analogous to that of Pomarol and Rattazzi, then the GUT physics does not decouple, in some cases leading to a viable spectrum [9].
} 
To summarize, we have found a model that has a gaugino spectrum generated purely from anomaly mediation, while the scalar spectrum results from summing two contributions to their squared masses: the pure anomaly-mediated contribution plus a gaugino-assisted contribution that looks like gauge mediation at the GUT scale. The latter can be positive and thus the slepton mass problem is solved.

\section{Spectrum and phenomenology}

We have seen that gaugino mediation without singlets yields a viable model with large contributions resulting from anomaly mediation. Since the gaugino masses are generated exclusively from anomaly mediation while the scalar masses have both the anomaly contributions as well as the gaugino-assisted contributions that we found above, the spectrum of the model has qualitative similarities with the phenomenological fix of adding a universal scalar mass squared to the anomaly-mediated contribution at the unification scale [26], which we call "AMSB $+m_{0}^{2}$ " scenario. However, it is clear from Eq. (3.17) that our "additional" scalar mass contribution is not universal, but instead proportional to a weighted sum over the quadratic Casimirs for the matter field of interest

$$
\tilde{m}_{i}^{2} \propto \sum_{a} C_{a}(i) g_{a}^{2} \quad a=U(1)_{Y}, S U(2)_{L}, S U(3)_{c} .
$$

In the case where the size of the extra dimension is of order the unification scale, the gauge couplings are the same and can be factorized out of the above. The gauginoassisted scalar mass contributions therefore differ merely by a sum over the quadratic Casimirs for each representation, which we show in Table [1.

Gaugino-assisted anomaly mediation has only a small number of input parameters in addition to those of the standard model, and is thus a highly predictive model of new physics. The anomaly-mediated contribution to soft terms is essentially dictated by a single unknown parameter, $m_{3 / 2}$. The gaugino-assisted contribution comes from the operators in Eq. (3.5)-(3.9) and are parameterized by unknown effective theory coefficients. Since the contributions from all of these operators are identical up to each of their order one coefficients, we parameterize their contributions by a single order one coupling $\eta$ which multiplies the quantity in Eq. (3.17). In addition, in the absence of unification, there are really three sets of operators with in principle differing coefficients, corresponding to the three gauge groups of the SM. We shall assume that gauge coupling unification is not an accident, and therefore the coupling is the same

for each gauge group. This is a necessary condition for embedding our model within a GUT group. 


\begin{tabular}{c|cc}
\hline \hline Representation & SM Casimir weight & $X$ and $Y$ gauge boson Casimir weight \\
\hline$Q$ & $21 / 10$ & $3 / 2$ \\
$u$ & $8 / 5$ & 2 \\
$d$ & $7 / 5$ & 1 \\
$L$ & $9 / 10$ & $3 / 2$ \\
$e$ & $3 / 5$ & 3 \\
$H_{u}$ & $9 / 10$ & $3 / 2$ \\
$H_{d}$ & $9 / 10$ & $3 / 2$ \\
\hline \hline
\end{tabular}

Table 1: The Casimir weights that enter the gaugino-assisted contribution to scalar masses resulting from five-dimensional loops with ordinary SM gauge fields (middle column) and $X$ and $Y$ gauge boson fields of SU(5) (right column), for each SM representation. Note that we have assumed $H_{u}$ and $H_{d}$ are in the fundamental representation of $\mathrm{SU}(5)$.

A third input of this model is the volume factor $M_{*} L$. We restrict ourselves to compactification scales which do not disrupt normal four-dimensional gauge coupling unification, i.e., we require $L^{-1} \gtrsim M_{G U T}$. This also means that we have no new proton decay problems beyond those of ordinary (four-dimensional) supersymmetric models. Using Eq. (2.1), this means there is an upper bound $M_{*} L \lesssim 22$. We are anyway interested in keeping $M_{*} L$ small so as not to introduce a new large hierarchy. However, as we explained in Sec. 2, avoiding bounds on FCNC requires $M_{*} L \gtrsim 16$, or equivalently, $L^{-1} \lesssim 2 M_{G U T}$.

Changing $M_{*} L$ affects the size of gaugino-assisted contribution, Eq. (3.17), but this model dependence can be equivalently absorbed in the coefficient $\eta$, and thus we can simply set $L^{-1}$ to $M_{\mathrm{GUT}}$ (if we ignore the small effects of the additional running). However, there can be an additional effect from decreasing $M_{*} L$, which is the turning on of contributions from GUT fields. Hence, we must distinguish between gauginoassisted anomaly mediation with or without GUT field contributions. In the case where we do have a GUT at the unification scale, we find that the GUT contributions are significant for the entire range of $L^{-1}$. In fact, for $L^{-1}$ near its upper limit of $2 M_{\mathrm{GUT}}$, gaugino-assisted contributions become nearly universal over complete GUT multiplets. Thus, for grand-unified gaugino-assisted anomaly mediation, an additional parameter $r=L^{-1} / M_{\mathrm{GUT}}$, with $1 \lesssim r \lesssim 2$, must be added. There is, of course, the additional issues of which GUT group, the representation that contains the Higgs, etc.

Finally, the remaining parameters are those of the Higgs sector. The magnitude of the supersymmetric mass parameter $\mu$ is fixed by the measured $Z$ boson mass while the soft parameter $B$ is unknown. We follow the usual practice and exploit the other 
electroweak symmetry breaking condition to trade the high scale parameter $B$ for the weak scale parameter $\tan \beta=\left\langle H_{u}\right\rangle /\left\langle H_{d}\right\rangle$.

Thus the model has the following free parameters:

$$
m_{3 / 2}, \eta, \tan \beta, \text { and } \operatorname{sign}(\mu)
$$

and also (at least) one more parameter, $r$, that enters if a GUT exists at the unification scale. We should emphasize that the above does not incorporate a specific mechanism to generate the $\mu$ term. As a result, we should also add the parameters $\delta m_{H_{u}}^{2}$ and $\delta m_{H_{d}}^{2}$ which are contributions to the Higgs soft parameters over and above those coming from AMSB and the gaugino-assisted contributions shown in Fig. 1. In principle, these additional Higgs mass contributions could result not just from a $\mu$-term mechanism (we discuss possible mechanisms to generate the $\mu$ term in the next section) but also from GUT threshold effects, e.g., our ignorance of the representation in which the Higgs field is embedded and/or the additional multiplets required for some version of doublet-triplet splitting. For simplicity, in the results we present below we shall assume that $\delta m_{H_{u}}^{2}$ and $\delta m_{H_{d}}^{2}$ are negligible, however a more thorough analysis of the parameter space is warranted.

Using the above set of input parameters, we can now compute the low energy spectrum. At the unification scale, the AMSB contribution to the scalar masses is summed with the gaugino-assisted contribution, and the entire set of renormalization group ( $\mathrm{RG}$ ) equations is evolved to the weak scale. We use full two-loop RG evolution in gauge couplings, Yukawa couplings, gaugino masses, scalar masses, and scalar trilinear couplings. In Fig. 2, we illustrate the running of soft masses with the input parameters $m_{3 / 2}=35 \mathrm{TeV}, \eta=1, \tan \beta=5$ and $\mu>0$, and without a GUT. Notice that the squarks are the heaviest sparticles of the spectrum, followed by the gluino, some combination of the Bino, sleptons, and Higgs, and finally the Wino as the LSP. These features are generic to most of the allowed parameter space. Since we are adding positive contributions to all of the sparticles, it is important to note that the up-type Higgs (mass) ${ }^{2}$ is negative, and therefore electroweak symmetry is broken radiatively as usual. The gaugino masses are precisely proportional to the SM beta functions, identical to the spectrum studied in the AMSB $+m_{0}^{2}$ scenario.

Now let's look at a spectrum assuming the existence of an SU(5) GUT. It is important to take into account the full gauge multiplet of the unified theory, as the additional gauge fields will now appear in the loops in Fig. 11. We compute this contribution in the Appendix for $r \geq 1$ in the case of an SU(5) GUT and present the results in Fig. 3 . Notice that the contributions are quite significant for both the left-handed and right- 


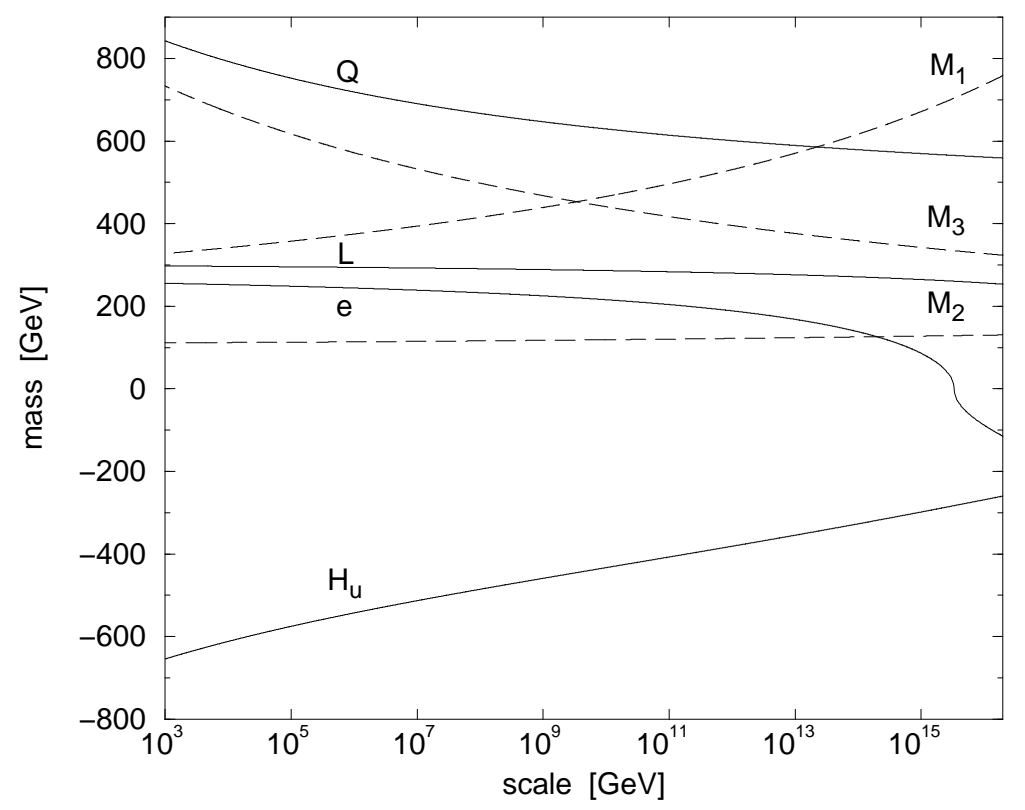

Figure 2: Soft masses as a function of scale. The input parameters are as follows: $m_{3 / 2}=35$ $\mathrm{TeV}, \eta=1, \tan \beta=5, \mu>0$, and $r=1$. We assume no new fields exist at the scale $M_{\mathrm{GUT}}$. For the gauginos (dashed) we plot $\left|M_{i}\right|$, and for scalars (solid) we plot $\operatorname{sign}\left(m^{2}\right) \sqrt{\left|m^{2}\right|}$.

handed sleptons. In fact, even at $r=1$ the contributions to the right-handed sleptons exceeds that from the standard model gauge multiplets.

To see the effects of the GUT on the spectrum, we run two sets of parameters, one with $m_{3 / 2}=35 \mathrm{TeV}, \eta=1, \tan \beta=5, \mu>0$ and $r=1$, and another with the same inputs except $\eta=1 / 2$ and $r=2$. The results are presented in Fig. 4 . Note the most significant change is that the left-handed sleptons are now the lightest scalar superpartners in all of our parameter space.

Another important consequence of the non-degeneracy of the gaugino-assisted scalar mass contribution is that the left-handed and right-handed slepton masses are no longer accidentally degenerate. It was first shown in Ref. [26] that the spectrum of AMSB with the phenomenological fix of adding a universal $m_{0}^{2}$ implies the weak scale masses $m_{\tilde{L}}$ and $m_{\tilde{e}}$ are the same to within a few percent. This is one of the few predictions that can also be used to distinguish this framework from generalized gauge mediation [37. In gaugino-assisted anomaly mediation, however, the gaugino-assisted contributions to the slepton masses differ by the ratio of their summed quadratic Casimirs. This can be read off from Table 1, $\delta m_{\tilde{L}}^{2} / \delta m_{\tilde{e}}^{2}=3 / 2$. Since the anomaly- 

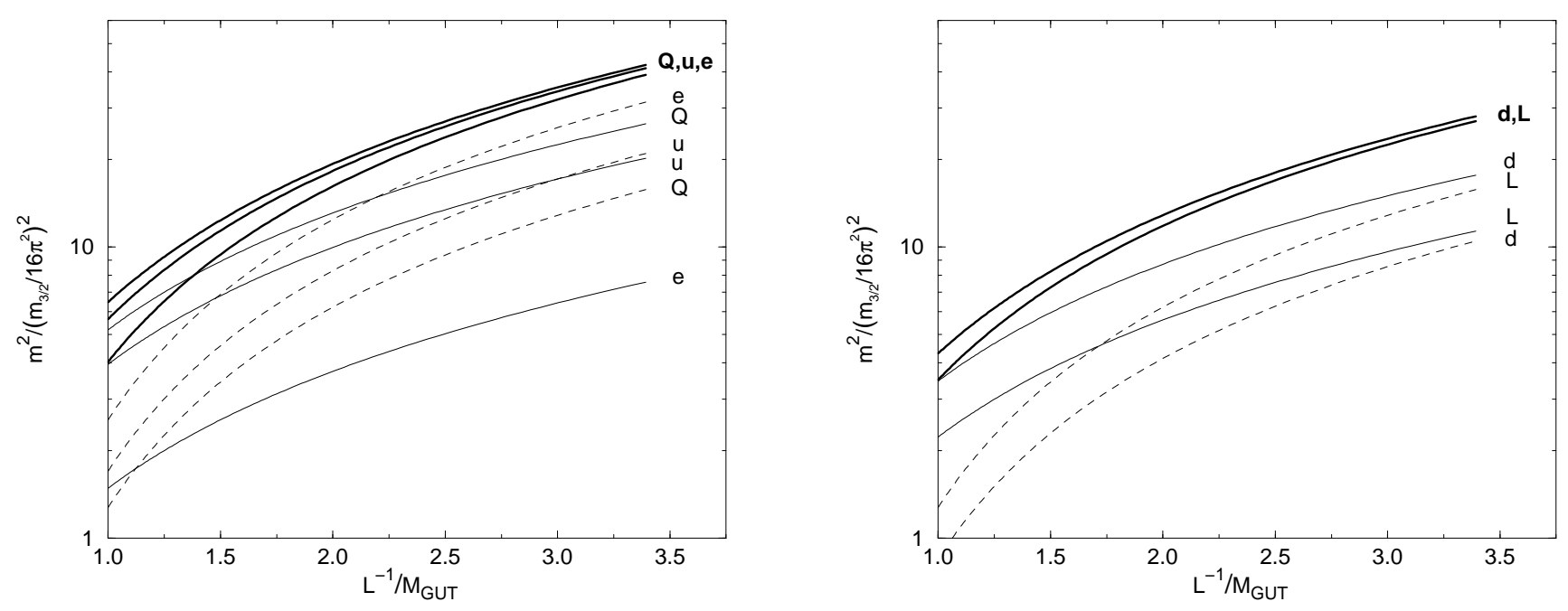

Figure 3: Gaugino-assisted contributions to squared scalar masses in units of $m_{3 / 2} / 16 \pi^{2}$. The thin solid lines indicate the contribution from $\mathrm{SU}(3) \times \mathrm{SU}(2) \times \mathrm{U}(1)$ gauge multiplets, the dashed lines represent contributions from $X$ and $Y$ gauge bosons and gauginos of the complete $\mathrm{SU}(5)$, and the thick solid lines are the sum of both contributions. For $r \gg 1$, the sums of these contributions converge for each complete multiplet $(Q, u$ and $e$ of the 10 and $d$ and $L$ of the $\overline{5}$.)

mediated contribution is also larger (but negative) for the left-handed slepton mass, one must combine the above with the anomaly-mediated contributions and evolve to the weak scale to determine the slepton mass spectra. In Fig. 5 we show the ratio of the left-handed to right-handed slepton masses at the weak scale as a function of $L^{-1} / M_{\text {unif }}$, both with and without heavy GUT field contributions. Note that, with or without a GUT, this ratio is nearly independent of the other parameters of the model, including the size of supersymmetry breaking $m_{3 / 2}$. When GUT field contributions are not present, we see that the left-handed slepton mass is always significantly larger than the right-handed slepton mass. Once GUT field contributions are included, the ratio is less than one. However, this last statement depends on the GUT group chosen. In fact, if we instead choose $\mathrm{SO}(10)$, the contributions to all chiral matter will be essentially universal (at large enough $r$ ) and the sleptons would be nearly degenerate, as occurs in the spectrum of AMSB $+m_{0}^{2}$ (though for very large $r$ one should take into account the renormalization group running above the GUT scale).

Since the gaugino masses are proportional to the SM beta functions, several analyses of the phenomenology and signals performed in the AMSB $+m_{0}^{2}$ scenario apply here 

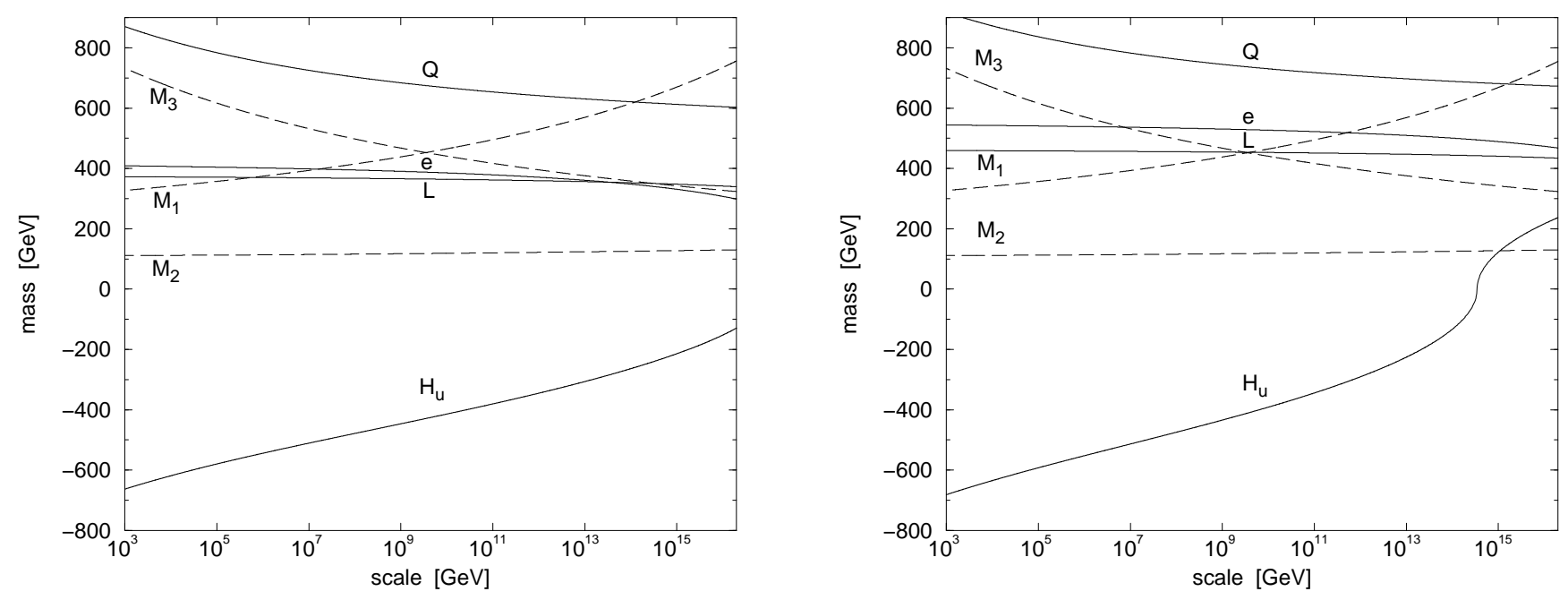

Figure 4: Soft masses as a function of scale. The input parameters for the figure on the left are the same as in Fig. 2 (with $r=1$ ). The figure on the right is the same except with $\eta=0.5$ and $r=2$. A minimal SU(5) GUT is assumed to exist at $M_{\mathrm{GUT}}$. As before, the gauginos (scalars) are the dashed (solid) lines.

as well [26, 38]. In particular, the lightest neutralino is mostly a Wino, with the lightest chargino nearly degenerate in mass. A careful calculation of this mass splitting was first done in [39]. For a Wino LSP, the near degeneracy results in a macroscopic decay length for the lightest chargino, typically of order a few centimeters [39, 40, 26] implying unique experimental signatures which have been analyzed by a number of groups [40, 26, 38, 37, 41]. In addition, the model also shares the cosmological features of the AMSB $+m_{0}^{2}$ scenario, including relaxing the cosmological problem associated with gravitino decay during nucleosynthesis, and the possibility of Wino LSP dark matter produced via non-thermal primordial gravitino decay [26, 42].

\section{Additional bulk fields and the $\mu$ term}

No model of supersymmetry breaking would be complete without a mechanism for generating the supersymmetric mass parameter $\mu$ for the superpotential operator $\mu H_{u} H_{d}$. As $\mu$ should be of the same order as soft masses, it is natural to assume that the $\mu$ term is generated by supersymmetry breaking.

There are several mechanisms on the market «, 7, 10, 11] specific to AMSB. However, maintaining the attractive features of the model restricts which mechanisms we 


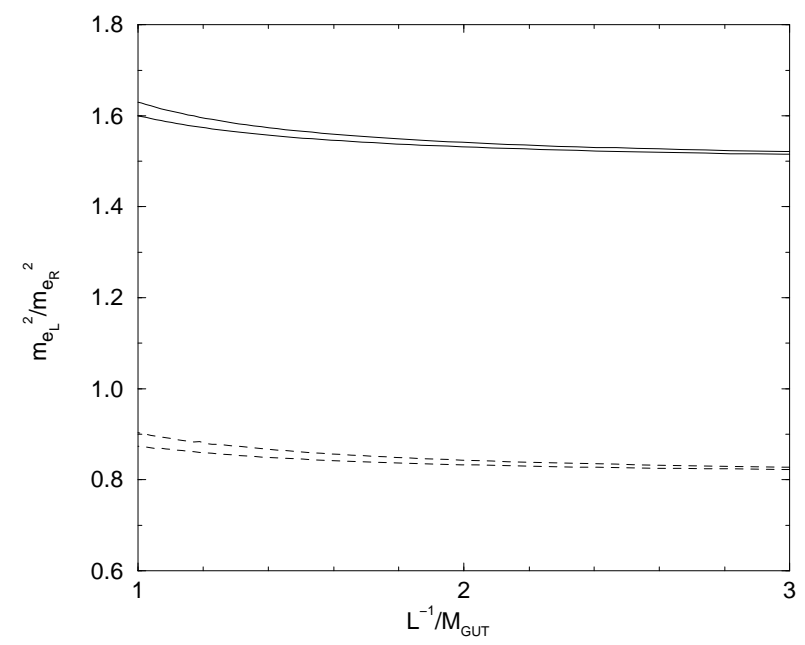

Figure 5: Ratio of the left-handed to right-handed slepton masses as a function of $L^{-1} / M_{\mathrm{GUT}}$ evaluated at the weak scale. The (upper, lower) solid lines correspond to models without GUT contributions, while the (lower, upper) dashed lines correspond to models with SU(5) GUT contributions (including thresholds), for $m_{3 / 2}=(35,70) \mathrm{TeV}$. Note that in the AMSB $+m_{0}^{2}$ scenario, the ratio is 1 to within a few percent [26], while in generalized gauge-mediation the ratio is significantly less than one 37.

can use. We discuss the restrictions, putting emphasis on the effects of adding bulk fields, and point out the class of mechanisms that work in our context.

The first requirement is that the Higgs fields live on the matter sector boundary. If the Higgs fields live in the bulk, their squared soft masses are expected to be of order $m^{2} \sim m_{3 / 2}^{2} \gg m_{\tilde{q}}^{2}$ due to direct couplings at the hidden sector boundary, and thus we avoid this scenario.

In addition, light bulk chiral superfields, Higgs or otherwise, can give significant enough contributions to flavor violation to encroach on current FCNC bounds. To see this, we introduce a bulk chiral superfield $B$. Now, the following operators can appear in the Lagrangian:

$$
\int d^{4} \theta \frac{\Sigma^{\dagger} \Sigma}{M_{*}^{3}} B^{\dagger} B \delta(y-L) \text { and } \int d^{4} \theta \frac{Q_{i}^{\dagger} Q_{j}}{M_{*}^{3}} B^{\dagger} B \delta(y)
$$

We can easily estimate $B$ 's one-loop contribution to squared scalar masses. It is proportional to $F_{\Sigma}^{2} / M_{*}^{6}$ and the effective cutoff of the loop integral is $L^{-1}$, so dimensional 
analysis gives us an estimate of the scalar mass contribution to be

$$
\delta m_{i j}^{2} \sim \frac{1}{16 \pi^{2}} \frac{F_{\Sigma}^{2}}{M_{*}^{2}} \frac{1}{\left(M_{*} L\right)^{4}} \sim \frac{1}{16 \pi^{2}} m_{3 / 2}^{2} \frac{1}{\left(M_{*} L\right)^{3}}
$$

which is only a volume factor suppressed when compared with the dominant scalar mass contribution. With coefficients of order one and numerical factors taken into account, this contribution is about an order of magnitude too large for $i, j=1,2$ [2]. One can either forbid new bulk chiral superfields or simply require that their couplings to boundary fields are $\lesssim \frac{1}{3} \|$, allowing $\mu$-term mechanisms such as "shining" [21, 30]. Vector multiplets in the bulk allow operators similar to those above but suppressed by additional powers of $M_{*}$. The additional volume suppression renders them harmless with respect to FCNC.

Viable mechanisms for producing the $\mu$ term that do not require additional bulk fields or contact interactions between the matter and hidden sectors appear in [7, 10]. These models require the existence of one or more standard model singlets to live on the matter boundary. For example, a $\mu$ (and $B \mu$ ) term of order the weak scale can be generated from an operator $H_{u} H_{d} X^{\dagger} / X$ in the Kähler potential. This operator gets a coefficient $\tilde{Z}\left(\sqrt{X X^{\dagger} / \Phi \Phi^{\dagger}}\right)$ from wave-function renormalization, where $\sqrt{X X^{\dagger} / \Phi \Phi^{\dagger}}$ should be taken as the renormalization scale and $X$ is the modulus-like field which has a large scalar vev but negligible auxiliary component. As shown in [7], this operator generates a $\mu$ term at one loop and a $B \mu$ term at two loops. The operator can be generated by a superpotential $\left[\lambda S H_{u} H_{d}+k S^{3}+y S^{2} X\right]$ (where $S$ is a singlet) and a kinetic mixing term between $X$ and $S$.

Finally, we comment on stabilization of the compactified dimension. As in anomaly mediation, the compact dimension can be stabilized by the mechanisms of [20] and [21]. However, a very recent analysis by Chacko and Luty 430 suggests that additional contributions to gaugino masses arise in the scenario of Ref. [20] from the radion multiplet if the gauge multiplets are in the bulk. The stabilization mechanism of [21] works in our models without significantly affecting the spectrum as long as one assumes slightly suppressed couplings of the new bulk fields to the boundaries as outlined above. It is an interesting prospect to see if this mechanism (or any new one) can be embedded into supergravity. We leave this speculation for possible future work.

\footnotetext{
"We note that in gaugino mediation [15, 16], these contributions are below experimental bounds as the flavor blind scalar masses are of order $\sim m_{3 / 2}$.
} 


\section{Conclusions}

We have presented a model of mediating supersymmetry breaking through an extra dimension. By putting standard model gauge fields and their superpartners in the bulk while requiring the hidden sector to be free of singlets, the gaugino spectrum is exactly that of anomaly-mediated supersymmetry breaking [4, 5]. The scalar masses obtain contributions from both anomaly mediation and operators which appear generically on the hidden-sector boundary. The latter amounts to non-supersymmetric contributions to wave-function renormalization of the gauge multiplet inducing scalar masses at the one-loop level. For order one couplings, the gaugino-assisted contribution is more than sufficient to make the squared masses of the sleptons positive.

The operators introduced in Sec. 3 induce a hard breaking of supersymmetry in

the four-dimensional effective theory below the scale $L^{-1}$. The hard breaking manifests itself as a tiny difference between the gauge and gaugino couplings to matter resulting in quadratically divergent contributions to scalars at one loop. We find that this way of calculating the contribution gives the same functional result as an explicit five-dimensional loop calculation. Furthermore, these operators should appear in any general four-dimensional effective theory with a hidden sector since no symmetries (including $R$ symmetries) are broken by them. The contributions to scalar masses from these operators are one-loop suppressed compared to those that come from contact terms. Thus, they are important when contact terms are absent, such as in models with sectors separated spatially in extra dimensions.

The phenomenology of the model contains some of the interesting features of the anomaly-mediated spectrum with a universal mass squared $m_{0}^{2}$ added to scalar fields [26]. In fact, if all standard model matter lives in a single multiplet of a grand unified theory, the contribution from gaugino-assisted anomaly mediation could be precisely a universal scalar mass. However, if you assume no new physics at the GUT scale, or a unified group like $\mathrm{SU}(5), \mathrm{SU}(6)$ or $\mathrm{SO}(10)$, the new contributions would not be universal, thus offering new spectra and thus new phenomenology. We therefore believe a more thorough analysis of this model would be of interest, as it is a new, highly predictive and very simple way of mediating supersymmetry breaking.

\section{Appendix: A GUT threshold calculation}

In this Appendix, we make explicit the calculation of gaugino-assisted contributions to scalar masses for compactification scales at or above the GUT scale. We assume there exists a unified theory and thus the loop contributions from additional gauge fields can 


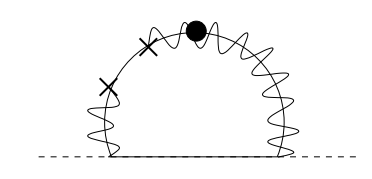

(a)

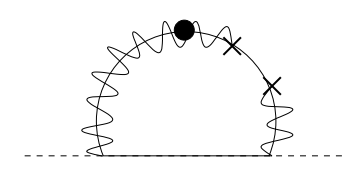

(b)

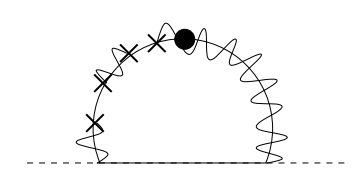

(c)

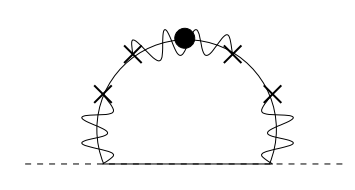

(d)

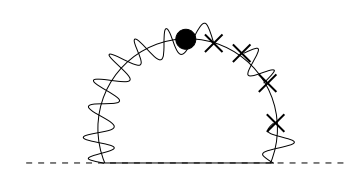

(e)

Figure 6: Loop contributions to squared scalar masses with $(\mathrm{a}, \mathrm{b})$ one and $(\mathrm{c}, \mathrm{d}, \mathrm{e})$ two insertions of $M_{\mathrm{GUT}}^{2} \times($ the adjoint propagator $)$.

be important. For concreteness, we assume a minimal SU(5) GUT and then discuss the model dependent and independent features of our results.

As discussed in the text, the compactification scale $L^{-1}$ cannot be too much larger than $M_{\mathrm{GUT}}$. Therefore, in calculating the contributions to scalar masses from $X$ and $Y$ bosons (and their superpartners), it will be important to include their order $M_{\mathrm{GUT}}$ masses. We use the method of mass insertions and then sum over loops with all number of insertions. For simplicity, we first present the calculation for a gauged U(1) broken at the GUT scale and later generalize to minimal SU(5) by summarizing the effects of the additional group structure.

We assume a field $\phi$ of charge +1 living on the matter boundary has a scalar vev $\langle\phi\rangle=M_{\mathrm{GUT}} /(\sqrt{2} g)$. To calculate the loop contribution to light scalar masses due to the $\mathrm{U}(1)$ vector multiplet and the operators (3.5)-(3.9), we must calculate diagrams like those in Fig. 1. In order to include the effects of the GUT masses, we include mass insertions on the gaugino propagators as in Fig. 6 where the crosses represent insertions of $M_{\mathrm{GUT}}$ and the solid lines in the upper part of the loop are propagators of $\psi$, the fermionic partner of $\phi$. The diagram in Fig. 6(a) has the functional form (at zero external momentum)

$$
\int \frac{d^{4} q}{(2 \pi)^{4}} \operatorname{tr}\left\{\sqrt{2} g_{(5)} P_{L}\left[\mathcal{P}(q ; 0,0) \sqrt{2} g_{(5)} \frac{M_{\mathrm{GUT}}}{\sqrt{2} g} \frac{-1}{\not q} \sqrt{2} g_{(5)} \frac{M_{\mathrm{GUT}}}{\sqrt{2} g}\right]\right.
$$




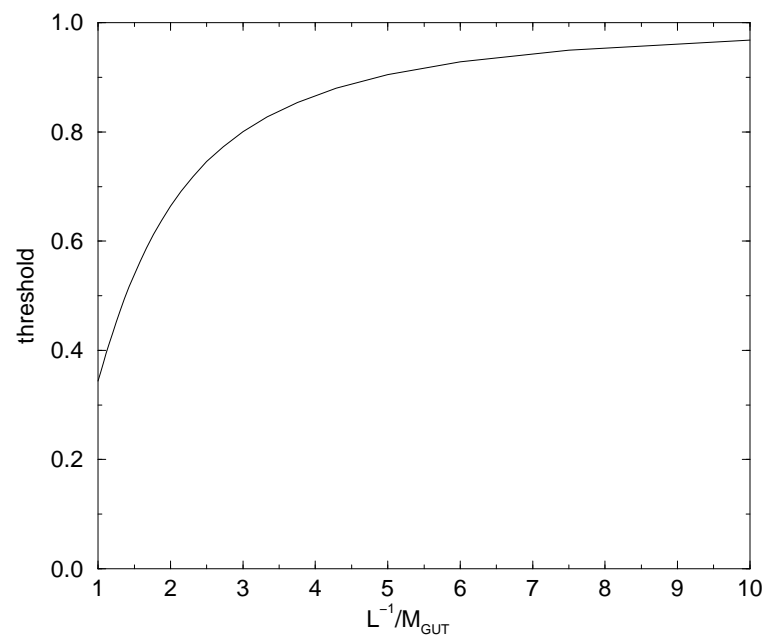

Figure 7: The squared scalar mass contribution from a GUT-scale gauge multiplet. The curve is normalized to the same contribution with $M_{\mathrm{GUT}}=0$.

$$
\left.\times \mathcal{P}(q ; 0, L) \frac{F^{2}}{M_{*}^{5}} \not \mathcal{P}(q ; L, 0) \sqrt{2} g_{(5)} \frac{-1}{\not q}\right\}
$$

where $g_{(5)}$ and $g$ are the five- and four-dimensional gauge couplings respectively, the quantity in square brackets represents the insertion on the gaugino propagator and the integral is done in Euclidean space. For the gaugino, we use the mixed position/momentum space propagator which appeared in [15:

$$
\begin{aligned}
\mathcal{P}(q ; a, b)= & \frac{2}{L} \sum_{m=0}^{\infty}\left[P_{L} \frac{1}{\sqrt{2} \delta_{n 0}} \cos \left(\frac{m \pi}{L} a\right)-P_{R} \sin \left(\frac{m \pi}{L} a\right)\right] \frac{\not-i \gamma_{5} \frac{m \pi}{L}}{q^{2}+\left(\frac{m \pi}{L}\right)^{2}} \\
& \times\left[P_{R} \frac{1}{\sqrt{2}} \cos \left(\frac{m \pi}{L} b\right)+P_{L} \sin \left(\frac{m \pi}{L} b\right)\right] .
\end{aligned}
$$

Since $\mathcal{P}(q ; 0,0)$ commutes with $1 / \not$, we see that Eq. (11) also represents Fig. 6(b).

Now we can generalize to any number of insertions. There are $n$ diagrams with $(n-1)$ insertions that have the form

$$
\int \frac{d^{4} q}{(2 \pi)^{4}} \operatorname{tr}\left\{2 g_{(5)}^{2} P_{L} \mathcal{P}(q ; 0, L) \frac{F^{2}}{M_{*}^{5}} \not \mathcal{P}(q ; L, 0) \frac{-1}{\not q}\left[\mathcal{P}(q ; 0,0) M_{\mathrm{GUT}}^{2} L \frac{-1}{\not d}\right]^{(n-1)}\right\}
$$

where we used the relation $g_{(5)}^{2}=g^{2} / L$. Using

$$
\sum_{n=1}^{\infty} n x^{n-1}=\frac{\partial}{\partial x} \sum_{n=1}^{\infty} x^{n}=\frac{\partial}{\partial x} \frac{1}{1-x}=\frac{1}{(1-x)^{2}}
$$


we sum over all GUT insertions and get

$$
\begin{aligned}
& \int \frac{d^{4} q}{(2 \pi)^{4}} \operatorname{tr}\left\{2 g_{(5)}^{2} P_{L} \mathcal{P}(q ; 0, L) \frac{F^{2}}{M_{*}^{5}} q \mathcal{P}(q ; L, 0) \frac{-1}{\not q} \frac{1}{\left[1+M_{\mathrm{GUT}}^{2} L \mathcal{P}(q ; 0,0) \frac{-1}{\mathscr{q}}\right]^{2}}\right\} \\
= & \int \frac{d^{4} q}{(2 \pi)^{4}} 4 g^{2} \frac{F^{2}}{M_{*}^{5} L} q^{2}\left(\sum_{m=0}^{\infty} \frac{1}{2^{\delta_{m 0}}} \frac{(-1)^{m}}{q^{2}+(m \pi / L)^{2}}\right)^{2} \frac{1}{\left[1-2 M_{\mathrm{GUT}}^{2} \sum_{m=0}^{\infty} \frac{1}{2^{\delta} m 0} \frac{1}{q^{2}+(m \pi / L)^{2}}\right]^{2}} \\
= & \frac{g^{2}}{2 \pi^{2}} \frac{F^{2}}{M_{*}^{5} L^{3}} \int d \tilde{q} \frac{\tilde{q}^{3}}{\sinh ^{2} \tilde{q}} \frac{1}{\left[1-\left(M_{\mathrm{GUT}} L\right)^{2}(\operatorname{coth} \tilde{q}) / \tilde{q}\right]^{2}}
\end{aligned}
$$

which can be computed numerically. In Fig. 7we have plotted the ratio of this integral to itself with $M_{\mathrm{GUT}}=0$ over a range of $\left(M_{\mathrm{GUT}} L\right)^{-1}$. We see that the non-zero masses of GUT fields should not be ignored for compactification scales up to a factor of a few times the GUT scale.

It is relatively straight forward to generalize the above result to a minimal $\mathrm{SU}(5)$ GUT. Take $\phi$ to now be in the adjoint representation with a vev

$$
\langle\phi\rangle=v_{\mathrm{GUT}}\left(\begin{array}{ccccc}
1 & & & & \\
& 1 & & & \\
& & 1 & & \\
& & & -\frac{3}{2} & \\
& & & & -\frac{3}{2}
\end{array}\right)=\sqrt{\frac{6}{5}} \frac{M_{\mathrm{GUT}}}{g} t^{24}
$$

where $t^{24}$ is a generator of $\mathrm{SU}(5)$ normalized such that $\operatorname{tr}\left[t^{24} t^{24}\right]=\frac{1}{2}$. Now the two quark-squark-gaugino vertices contribute the factor $2 g^{2}\left(t^{a} t^{b}\right)_{j}^{i}$. The insertions of $M_{\mathrm{GUT}}$ now come with structure constants $f^{24 a c}$. In the normalization we chose, $\sum_{c}\left(f^{24 a c} f^{24 b c}\right)=\sqrt{5 / 12} \delta_{a b}^{\prime}$, where $\delta^{\prime}=1$ when $a=b=x$ with $x$ representing the broken generators $\mathrm{SU}(5) /[\mathrm{SU}(3) \times \mathrm{SU}(2) \times \mathrm{U}(1)]$, and $\delta^{\prime}=0$ otherwise. This change gives us the same $M_{\mathrm{GUT}} / \sqrt{2} g$ insertions as before. The only difference from the Abelian case is a "partial Casimir" which depends on the representation (see Table 1 in Sec. (1) multiplying the integral.

In the calculation done above, all additional GUT fields or interactions have been ignored. For instance, in order to get a vev, $\phi$ must appear in superpotential terms, the simplest being a Majorana mass term. These new interactions can have an effect the threshold calculation. The most significant of which could come from the mechanism which splits the Higgs doublets from the rest of their multiplet ("doublet-triplet splitting"). The new multiplets required for such a mechanism could also contribute to Higgs soft masses at two loops if the new representations are large enough. Finally, 
other GUT groups will obviously have different contributions. For instance, at $r=2$, the gaugino-assisted contribution in minimal SO(10) would be approximately universal for all chiral matter. The group $E_{6}$ could potentially have a universal contribution to all matter fields. However, realistically we expect things like doublet-triplet splitting and contributions from $D$ terms to destroy this naive degeneracy.

\section{Acknowledgments}

We are grateful to Zackaria Chacko, Hsin-Chia Cheng, Markus Luty, Erich Poppitz, Martin Schmaltz, Yael Shadmi, and Carlos Wagner for many useful discussions. In addition, we thank Hsin-Chia Cheng and Tim Tait for comments on the final draft. We also thank Zackaria Chacko and Markus Luty for communicating some of the results of their paper Ref. 43 to us before it was completed. GDK would like to thank the Argonne high energy theory group and the organizers of the Argonne Theory Institute 2000 for their hospitality. We also thank the Aspen Center for Physics where this work was completed. DEK is supported in part by the DOE under contracts DE-FG0290ER40560 and W-31-109-ENG-38. GDK is supported in part by the DOE under contracts DOE-ER-40682-143 and DE-FG02-95ER40896.

\section{References}

[1] Y. Nir, N. Seiberg, Phys. Lett. B309 (1993) 337 [hep-ph/9304307];

M. Dine, R. Leigh, A. Kagan, Phys. Rev. D48 (1993) 4269 [hep-ph/9304299];

[2] For a review, see F. Gabbiani, E. Gabrielli, A. Masiero, L. Silvestrini, Nucl. Phys. B477 (96) 321 [hep-ph/9604387].

[3] M. Dine, A.E. Nelson, Phys. Rev. D48 (1993) 1277 [hep-ph/9303230]; M. Dine, A.E. Nelson, Y. Shirman, Phys. Rev. D51 (1995) 1362 [hep-ph/9408384]; M. Dine, A.E. Nelson, Y. Nir, Y. Shirman, Phys. Rev. D53 (1996) 2658 [hep-ph/9507378]. For a review, see G.F. Giudice, R. Rattazzi Phys. Rep. 322 (1999) 419 [hep-ph/9801271].

[4] L. Randall, R. Sundrum, Nucl. Phys. B557 (1999) 79 [hep-th/9810155].

[5] G.F. Giudice, M.A. Luty, H. Murayama, R. Rattazzi, JHEP 9812:027 (1998) [hep-ph/9810442].

[6] J.A. Bagger, T. Moroi, E. Poppitz, JHEP 0004:009 (2000) [hep-th/9911029].

[7] A. Pomarol, R. Rattazzi, JHEP 9905:013 (1999) [hep-ph/9903448]. 
[8] R. Rattazzi, A. Strumia, J.D. Wells, Nucl. Phys. B576 (2000) 3 [hep-ph/9912390].

[9] Z. Chacko, M. Luty, E. Pontón, Y. Shadmi, Y. Shirman, [hep-ph/0006047].

[10] E. Katz, Y. Shadmi, Y. Shirman, JHEP 9908:015 (1999) [hep-ph/9906296].

[11] Z. Chacko, M.A. Luty, I. Maksymsk, E. Pontón, JHEP 0004:001 (2000) [hep-ph/9905390].

[12] I. Jack, D.R.T. Jones, Phys. Lett. B482 (2000) 167 [hep-ph/0003081]; [hep-ph/0006116].

[13] M. Carena, K. Huitu, T. Kobayashi, [hep-ph/0003187].

[14] Z. Chacko, M.A. Luty, E. E. Pontón, JHEP 0007:036 (2000) [hep-ph/9909248]; Y. Nomura, T. Yanagida, [hep-ph/0005211].

[15] D.E. Kaplan, G.D. Kribs, M. Schmaltz, Phys. Rev. D62 (2000) 035010 [hep-ph/9911293].

[16] Z. Chacko, M. Luty, A.E. Nelson, E. Pontón, JHEP 0001:003 (2000) [hep-ph/9911323].

[17] I. Affleck, M. Dine, N. Seiberg, Nucl. Phys. B256 (1985) 557.

[18] M. Dine, D. MacIntire, Phys. Rev. D46 (1992) 2594.

[19] M. K. Gaillard, B. Nelson, Y. Wu, Phys. Lett. B459 (1999) 549 [hep-th/9905122]; M. K. Gaillard, B. Nelson, [hep-th/0004170].

[20] M.A. Luty, R. Sundrum, Phys. Rev. D62 (2000) 035008 [hep-th/9910202].

[21] N. Arkani-Hamed, L. Hall, D. Smith, N. Weiner, [hep-ph/9911421].

[22] N. Arkani-Hamed, S. Dimopoulos, G. Dvali, Phys. Lett. B429 (1998) 263 [hep-ph/9803315]; Phys. Rev. D59 (1999) 086004 [hep-ph/9807344].

[23] E. Cremmer, B. Julia, J. Scherk, S. Ferrara, L. Girardello, P. van Nieuwenhuizen, Nucl. Phys. B147 (1979) 105; E. Cremmer, S. Ferrara, L. Girardello, A. Van Proeyen, Nucl. Phys. B212 (1983) 413.

[24] G.F. Giudice, R. Rattazzi, Nucl. Phys. B511 (1998) 25 [hep-ph/9706540];

N. Arkani-Hamed, G.F. Giudice, M.A. Luty, R. Rattazzi Phys. Rev. D58 (1998) 115005 [hep-ph/9803290].

[25] I. Jack, D.R.T. Jones, Phys. Lett. B465 (1999) 148 [hep-ph/9907255]. 
[26] T. Gherghetta, G.F. Giudice, J.D. Wells, Nucl. Phys. B559 (1999) 27 [hep-ph/9904378].

[27] J. Feng, T. Moroi, Phys. Rev. D61 (2000) 095004 [hep-ph/9907319].

[28] E.A. Mirabelli, M.E. Peskin, Phys. Rev. D58 (1998) 065002 [hep-th/9712214].

[29] J. Ellis, C. Kounnas, D.V. Nanopoulos, Nucl. Phys. B247 (1984) 373; A.B. Lahanas, D.V. Nanopoulos, Phys. Rep. 145 (1987) 1.

[30] M. Schmaltz, W. Skiba, [hep-ph/0001172].

[31] M. Schmaltz, W. Skiba, [hep-ph/0004210].

[32] D.E. Kaplan, T. Tait, JHEP 0006:020 (2000) [hep-ph/0004200].

[33] H.-C. Cheng, J.L. Feng, N. Polonsky, Phys. Rev. D56 (1997) 6875 [hep-ph/9706438];

E. Katz, L. Randall, S. Su, Nucl. Phys. B536 (1998) 3 [hep-ph/9801416].

[34] N. Arkani-Hamed, Y. Grossman, M. Schmaltz, [hep-ph/9909411].

[35] E. Poppitz, S.P. Trivedi, Phys. Lett. B401 (1997) 38 [hep-ph/9703246].

[36] G.D. Kribs, Nucl. Phys. B535 (1998) 41 [hep-ph/9803259].

[37] G.D. Kribs, Phys. Rev. D62 (2000) 015008 [hep-ph/9909376].

[38] J.L. Feng, T. Moroi, Phys. Rev. D61 (2000) 095004 [hep-ph/9907319].

[39] H.-C. Cheng, B.A. Dobrescu, K.T. Matchev, Nucl. Phys. B543 (1999) 47 [hep-ph/9811316].

[40] J.L. Feng, T. Moroi, L. Randall, M. Strassler, S. Su, Phys. Rev. Lett. 83 (1999) 1731 [hep-ph/9904250].

[41] J. F. Gunion, S. Mrenna, Phys. Rev. D62 (2000) 015002 [hep-ph/9906270]; S. Su, Nucl. Phys. B573 (2000) 87 [hep-ph/9910481]; F. E. Paige, J. Wells, [hep-ph/0001249]; D. K. Ghosh, P. Roy and S. Roy, JHEP 0008:031 (2000) [hep-ph/0004127]; H. Baer, J. K. Mizukoshi and X. Tata, Phys. Lett. B488 (2000) 367 [hep-ph/0007073].

[42] T. Moroi, L. Randall, Nucl. Phys. B570 (2000) 455 [hep-ph/9906527].

[43] Z. Chacko, M. Luty, [hep-ph/0008103]. 